\title{
High resistance to fourth-generation cephalosporins among clinical isolates of Enterobacteriaceae producing extended- spectrum $\beta$-lactamases isolated in Portugal
}

\author{
Ruben Fernandes \\ Álvaro Gestoso \\ José Mota Freitas \\ Perpétua Santos \\ Cristina Prudêncio*
}

\begin{abstract}
Ciências Químicas e das Biomoléculas, Escola Superior de Tecnologia da Saúde do Porto, Instituto Politécnico do Porto, Portugal

* Corresponding author. Present address: Ciências Químicas e das Biomoléculas, Praça do Coronel Pacheco, $\mathrm{n}^{\circ} 15,4050$ Porto, Portugal. Tel.: +351 22206 1004; fax: +35122 2061001.

E-mail address: cps@estsp.ipp.pt (C. Prudêncio)
\end{abstract}

Sir,

Here we report the molecular and antimicrobial susceptibility profile of extended-spectrum $\beta$-lactamase (ESBL)-producing strains found in the Portuguese northern occidental coast region (Minho). For this purpose, bacteria isolated from clinical hospitalised and non-hospitalised patients over a period of 2 years were identified and minimal inhibitory concentrations (MICs) were determined by microdilution methods according to the Clinical and Laboratory Standards Institute (formerly the National Committee for Clinical Laboratory Standards) guidelines on Enterobacteriaceae. Additionally, ESBL phenotypic identification was confirmed by the Etest (AB BIODISK, Solna, Sweden). Various methods of molecular identification of the $\beta$-lactamase (bla) genes, involving polymerase chain reaction (PCR) and sequencing strategies, were used in this study.

The ESBL-producing strains $(n=193)$ were isolated from urine ( $n=127)$, sputum $(n=42)$, bronchoalveolar lavage $(n=14)$, blood $(n=7)$ and ascitic fluid $(n=3)$. The most frequent ESBL-producing organism isolated in the present study was Escherichia coli $(67.9 \%$; $n=131$ ), followed by Klebsiella pneumoniae (30.6\%; $n=59$ ), Klebsiella oxytoca $(0.5 \% ; n=1)$, Enterobacter aerogenes $(0.5 \% ; n=1)$ and Citrobacter freundii $(0.5 \% ; n=1)$. The ESBL detected in the present study were the TEM type (40.4\%), CTX-M type (36.8\%) and SHV type (22.8\%).

TEM-52 and TEM-24 were the most frequent TEM types $(20.2 \%$ and $12.9 \%$, respectively). Members of TEM-10 (4.1\%) and TEM-116 (2.1\%) were also detected.

Within the CTX-M family, CTX-M-9 group was represented by CTX-M-9 (13.5\%) and CTX-M-14 (8.4\%). In the CTX-M-1 group, CTX$\mathrm{M}-15$ was the most frequent type (12.4\%), followed by CTX-M-1 (2.1\%), CTX-M-3 (0.5\%) and CTX-M-32 (0.5\%). Regarding CTX-M types, it appears that CTX-M-14 is widespread among the northwestern Iberian Peninsula [1]. Klebsiella pneumoniae harbouring a CTX-M-15 enzyme was described for the first time in Portugal in 2005 [2] in the Lisbon area, but CTX-M-15 enzyme has also recently been found by us in the north of Portugal in another Enterobacteriaceae member, isolated from bloodstream infections [3] among seven patients in two different hospitals. Other ESBL-producing species (not E. coli or K. pneumoniae) were also found. This is the first time that $C$. freundii has been described as a producer of CTX-M-32 in this country.

The SHV enzymes occurred only in $23.3 \%$ of all ESBL-producing organisms. Within this type, the most frequent type was SHV-12 (12.4\%), followed by SHV-5 (8.8\%) and finally SHV-2 (2.1\%).

Some isolates co-produced more than one ESBL type: TEM-52/CTX-M-14 (0.5\%); TEM-116/CTX-M-14 (0.5\%); and TEM116/CTX-M-15 (0.5\%).

MIC testing showed that isolates producing ESBLs were mostly susceptible to carbapenems (100\%) and amikacin (99.5\%). In con- trast, ESBL-producing strains presented low susceptibility rates to cefepime and quinolones. Indeed, $98.9 \%$ of the ESBL-producing strains were cefepime-resistant and $85.4 \%$ were resistant to quinolones (ciprofloxacin and norfloxacin). In the generality, these high levels of resistance to quinolones were more conspicuous in members of the CTX-M family (98.1\%) than TEM and SHV types (80.8\% and $72.1 \%$, respectively).

In this study, cefepime presented a surprisingly low activity against ESBL-producing microorganisms. Recent literature refers to the inoculum effect exhibited by cefepime [4]. Nevertheless, we believe that this should not be pointed out as a single explanation once MIC determination is performed using inoculum concentrations of 0.5 McFarland standard. In our sample, only two $K$. pneumoniae harbouring SHV-2 ESBL were susceptible to cefepime. All the other clinical isolates (98.9\%) expressing the ESBL phenotype were resistant to cefepime. It seems interesting that a recent study showed that cefepime was successfully administered to three patients (two females and one male) aged between 47 years and 87 years carrying a Gram-negative ESBL-positive strain [5]. Nevertheless, other studies worldwide have begun to describe the emergence of high resistance to cefepime among Gram-negative ESBL-producers [6].

The present work showed a high diversity of ESBL enzymes occurring in the north of Portugal. In this country, the most prevalent type is still the TEM type, but CTX-M is growing rapidly [7]. The emergence of ESBL-producers resistant to cefepime in Portugal is a matter of concern. We believe that the uncontrolled use of cephalosporins may have an important role in the acquisition of resistance mechanisms, particularly the production of ESBL enzymes. Establishment of policies to monitor drug delivery in hospital and ambulatory pharmacies as well as implementation of public health defence strategies towards health promotion and drug resistance prevention appear to be urgent.

Funding: From PhD fees attributed to Dr. Ruben Fernandes.

Competing interests: None declared.

Ethical approval: Not required.

\section{References}

[1] Livermore DM, Canton R, Gniadkowski M, Nordmann P, Rossolini GM, Arlet G, et al. CTX-M: changing the face of ESBLs in Europe. J Antimicrob Chemother 2007;59:165-74.

[2] Conceição T, Brízio A, Duarte A, Lito LM, Cristino JM, Salgado MJ. First description of CTX-M-15-producing Klebsiella pneumoniae in Portugal. Antimicrob Agents Chemother 2005;49:477-8.

[3] Fernandes R, Vieira M, Ferraz R, Prudêncio C. Bloodstream infections caused by multidrug-resistant Enterobacteriaceae: report from two Portuguese hospitals. J Hosp Infect 2008;70:93-5.

[4] Queenan AM, Foleno B, Gownley C, Wira E, Bush K. Effects of inoculum and beta-lactamase activity in AmpC- and extended-spectrum beta-lactamase (ESBL)-producing Escherichia coli and Klebsiella pneumoniae clinical isolates tested by using NCCLS ESBL methodology. J Clin Microbiol 2004;42:269-75.

[5] Bhavnani SM, Ambrose PG, Craig WA, Dudley MN, Jones RN. SENTRY Antimicrobial Surveillance Program Outcomes evaluation of patients with ESBL- and non-ESBL-producing Escherichia coli and Klebsiella species as defined by CLSI reference methods: report from the SENTRY Antimicrobial Surveillance Program. Diagn Microbiol Infect Dis 2006;54:231-6.

[6] Grover SS, Sharma M, Chattopadhya D, Kapoor H, Pasha ST, Singh G. Phenotypic and genotypic detection of ESBL mediated cephalosporin resistance in Klebsiella pneumoniae: emergence of high resistance against cefepime, the fourth generation cephalosporin. J Infect 2006;53:279-88.

[7] Hernández JR, Martínez-Martínez L, Cantón R, Coque TM, Pascual A. Spanish Group for Nosocomial Infections (GEIH), Nationwide study of Escherichia coli and Klebsiella pneumoniae producing extended-spectrum beta-lactamases in Spain. Antimicrob Agents Chemother 2005;49:2122-5. 